•研究报告・

\title{
青藏高原高山区和泛北极地区种子植物多倍体比较
}

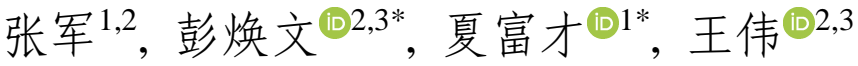

1. 北华大学林学院, 吉林吉林 132013; 2. 中国科学院植物研究所系统与进化植物学国家重点实验室, 北京 100093; 3. 中国科学院大学, 北 京 100049

摘要：多倍化是植物快速适应极端环境胁迫的一种重要机制。青藏高原高山区和泛北极地区具有相似的极端低温环境，且两 地的植物曾有密切的交流和联系。然而, 多倍体物种对两地植物区系生物多样性的贡献是否相同仍不清楚。我们系统地收集 两地已有染色体数目和倍性报道的种子植物物种名录, 共计1,770种, 其中青藏高原高山区774种, 泛北极地区996种; 同时也 相应地收集了每个物种的生活型信息。分析显示青藏高原高山区多倍体植物的比例为 $20.9 \%$, 泛北极地区多倍体植物比例为 $61.5 \%$; 青藏高原高山区一年生草本、多年生草本和木本植物中多倍体的比例分别为 $20.7 \% 、 21.6 \%$ 和 $12.8 \%$, 泛北极地区一年 生草本、多年生草本和木本植物中多倍体的比例分别为 $60.2 \% 、 65.5 \%$ 和 $38.3 \%$ 。这些结果表明泛北极地区比青藏高原高山区 具有较高比例的多倍体物种。青藏高原高山植物区系在渐新世-中新世之交开始兴起, 此时高原已达到一定高度, 而后的高寒 环境相对稳定，致使多倍体物种相对较少; 而泛北极地区植物区系在3-4 Ma兴起，此后经历了冰期-间冰期、海平面波动等反 复剧烈的气候环境变化, 可能促进了大量的多倍化事件发生。本研究通过比较青藏高原高山区和泛北极地区植物多倍体物种 的比例, 揭示了两地多倍体比例差异的可能原因, 将提高对多倍体适应极端环境的理解。

关键词: 青藏高原; 泛北极地区; 多倍化; 生活型; 极端生境; 气候变化

张军, 彭焕文, 夏富才, 王伟 (2021) 青藏高原高山区和泛北极地区种子植物多倍体比较. 生物多样性, 29, 1470-1480. doi: 10.17520/biods.2021146. Zhang J, Peng HW, Xia FC, Wang W (2021) A comparison of seed plants' polyploids between the Qinghai-Tibet Plateau alpine and the Pan-Arctic regions. Biodiversity Science, 29, 1470-1480. doi: 10.17520/biods.2021146.

\section{A comparison of seed plants' polyploids between the Qinghai-Tibet Plateau alpine and the Pan-Arctic regions}

Jun Zhang ${ }^{1,2}$, Huanwen Peng ${ }^{\left(\mathbb{D}_{2,3 *}\right.}$, Fucai Xia ${ }^{\mathbb{D}_{1 *}}$, Wei Wang ${ }^{\left(\mathbb{D}_{2,3}\right.}$

1 Forestry College, Beihua University, Jilin, Jilin 132013

2 State Key Laboratory of Systematic and Evolutionary Botany, Institute of Botany, Chinese Academy of Sciences, Beijing 100093

3 University of the Chinese Academy of Sciences, Beijing 100049

\section{ABSTRACT}

Aims: Polyploidization is an important mechanism for plants to adapt to extreme environments. The Qinghai-Tibet Plateau (QTP) alpine and Pan-Arctic regions have a similar low-temperature environment, and there were closely biotic exchanges between the two regions. However, it is unclear whether the QTP alpine and Pan-Arctic floras have similar polyploid buildup or not. Here, we compared polyploid proportions between these two floras and explored the potential causes for the difference.

Methods: By comprehensive searches in the databases and various literatures, we obtained species lists with chromosome numbers and ploidy of seed plants for the QTP alpine and Pan-Arctic regions, and then calculated polyploid proportion in totality as well as polyploid proportion for different life forms in the two regions.

Results: A total of 1,770 species of seed plants with chromosome numbers were collected, of which 774 occur in the QTP alpine region and 996 occur in the Pan-Arctic. According to the statistical analyses, the proportions of polyploid plants are 20.9\% in the QTP alpine region and 61.5\% in the Pan-Arctic. The proportions of polyploids of annual herbs, perennial herbs and woody plants in the QTP alpine region are $20.7 \%, 21.6 \%$, and $12.8 \%$, respectively. The proportions

收稿日期: 2021-04-18; 接受日期: 2021-07-03

基金项目：中国科学院战略性科技先导专项(B类) (XDB31030000)和国家自然科学基金(31770233; 31770231; 32011530072)

* 共同通讯作者 Co-authors for correspondence. E-mail: penghuanwen@ibcas.ac.cn; xfc0707@163.com 
of polyploids of annual herbs, perennial herbs and woody plants in the Pan-Arctic are $60.2 \%$, 65.5\%, and 38.3\%, respectively.

Conclusions: The polyploid proportions in totality and for different life forms in the Pan-Arctic are higher than those in the QTP alpine region obviously, which is associated with the different evolutionary history of the two floras, as well as their different geological and climatic events. The modernization of the QTP alpine flora took place around the Oligocene-Miocene boundary, and since then the QTP has had a relatively constant low-temperate environment, whereas the Pan-Arctic flora did not develop until 3-4 Ma, and since then the Pan-Arctic flora experienced repeated glacial and interglacial periods and repeated sea-level fluctuations, which might have resulted in the polyploidization of plants. This study contributes to our knowledge on how polyploids adapt to low-temperate environments.

Key words: Qinghai-Tibet Plateau; Pan-Arctic; polyploidization; life form; extreme environments; climate change

多倍体(polyploid)是指体细胞中含有3个或3个 以上染色体组的个体(Bennett, 2004)。多倍体在植物 中广泛存在, 在植物物种多样性中占有较大比例。 如，现存被子植物中约有70\%的物种在其进化史上 发生过一次或多次多倍化事件(Masterson, 1994)。外 界极端环境胁迫可能导致未减数配子形成频率的 增加, 从而促使多倍体的形成(De Storme \& Geelen, 2014)。已有研究表明多倍化事件的发生与环境胁迫 密切相关, 多倍化能提高生物快速适应极端环境变 化的能力(Cai et al, 2019; Van de Peer et al, 2021)。低 温是极端环境的一种, 如高山或极地地区。这些区 域对全球气候变暖更为敏感, 其生物多样性受影响 更大。Wu等(2020)通过对被子植物古多倍化的研究, 发现多倍化有助于植物适应白严纪-古新世交界时 期(Cretaceous-Paleocene boundary)的寒冷、黑暗等 剧烈变化的极端环境。多倍化已被认为是适应低温 的一种重要机制(Levin, 2002)。但在不同低温环境 中, 多倍体植物的比例或者对其植物区系生物多样 性的贡献是否相同, 尚缺乏比较研究。

青藏高原高山区和泛北极地区是地球上具有 相似的极端低温环境的陆地生态系统, 它们孕育了 北半球两个面积最大、范围最广的低温植物区系 (Gao et al, 2016; Gamberg et al, 2020)。它们不仅具 有相似的低温环境, 而且植物区系在历史上也存在 着较为密切的联系。分子系统学研究表明青藏高原 高山区是泛北极地区植物区系生物多样性的一个 重要起源地(如: Li et al, 2014; Zhang et al, 2014; Favre et al, 2016; Wang et al, 2016), 也有个别类群 起源于北极而后南迁至青藏高原, 如岩须属 (Cassiope)植物(Hou et al, 2016)。随着全球气候变化 与人类活动的日益加剧, 两地的平均气温快速升高, 低温生境急剧缩减(Liu \& Chen, 2000; Serreze \&
Barry, 2011), 这将致使两地喜冷植物面临分布区消 失的风险。两地生物多样性正面临严重威胁, 尤其 是青藏高原针叶林林线上移以及泛北极地区灌木 类群分布区的扩张, 可能将进一步加速生物多样性 的丧失(Myers-Smith et al, 2015; Wang et al, 2019)。 生物多样性的形成和维持机制是生态学和进化生 物学领域共同关注的科学问题(Zhang et al, 2014; Gao et al, 2016)。比较研究多倍体在两地植物区系 组成中所占比例的差异, 不仅有助于理解两地生物 多样性的起源, 而且能增进对多倍体适应极端低温 环境机制的理解。

青藏高原位于亚洲中部, 平均海拔在 $4,000 \mathrm{~m}$ 以上, 有世界“第三极”之称。本研究采用广义青藏高 原的范围, 包括青藏高原、横断山和喜马拉雅山 (Spicer et al, 2021), 即西起帕米尔高原, 东至横断 山脉东部, 南至喜马拉雅山脉南缘, 北以昆仑山一 祁连山北侧为界, 总面积约 $2.5 \times 10^{6} \mathrm{~km}^{2}$ (图1; 张 镱锂等, 2002; Zhang et al, 2016)。根据Körner (2003), 我们所采用的青藏高原高山区的范围包括林线以 上的区域。高原内部山脉、沟谷纵横, 生境类型丰 富多样, 孕育了极高的生物多样性和特有性, 有种 子植物 1,619 属 12,000 余种, 其中 $38 \%$ 为该地区特有 种(潘保田等, 2004; Zhang et al, 2016)。泛北极地区 被亚洲、欧洲以及北美洲环抱，其边界与亚、欧、 北美三大洲针叶林北部边界重合(图1; Elven et al, 2011), 区内分布着北半球纬度最高的植物区系, 有 种子植物2,146种(Elven et al, 2011)。青藏高原高山 区和泛北极地区都具有极端低温环境, 但两地具有 明显不同的地质历史。受印度次大陆和欧亚板块碰 撞的影响, 青藏高原的地形、地貌自新生代以来发 生了很大变化(Yuan et al, 2021), 尤其自渐新世-中 新世之交, 青藏高原中部、东北部发生了显著隆升, 


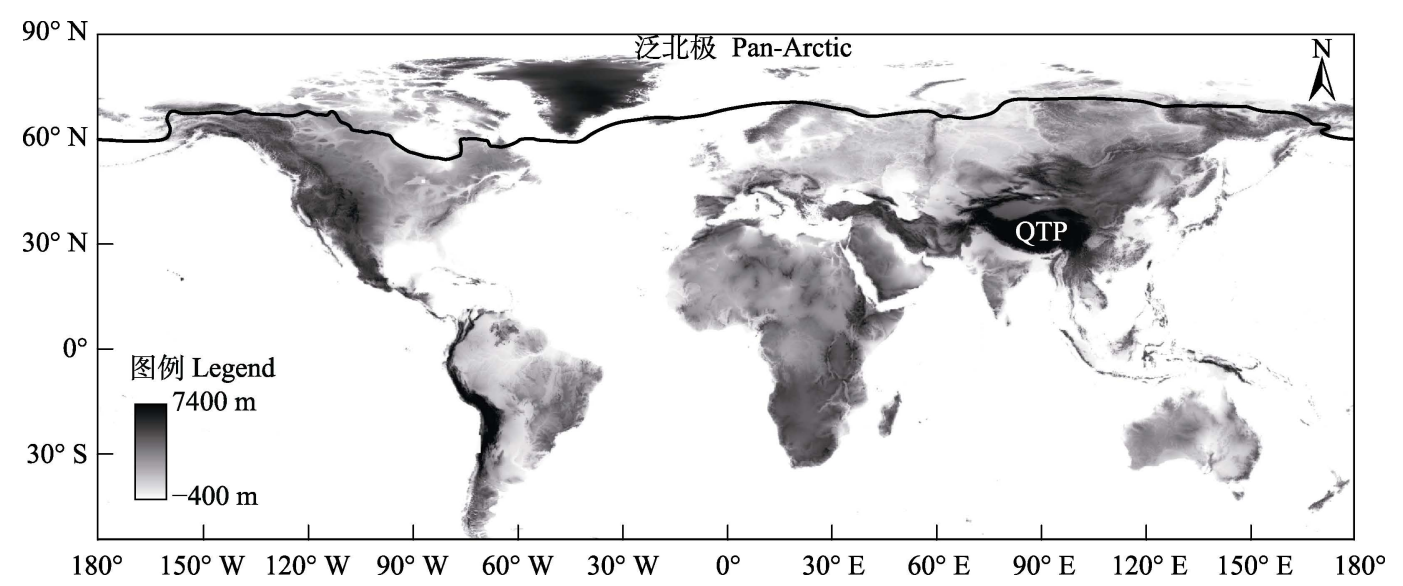

图1 青藏高原(QTP)和泛北极地区范围。青藏高原范围依据张镱锂等(2002)和Zhang等(2016), 泛北极地区范围依据Elven等 (2011)。

Fig. 1 Ranges of the Qinghai-Tibet Plateau (QTP) and the Pan-Arctic regions. The range of the QTP refers to Zhang YL et al (2002) and Zhang DC et al (2016); the range of the Pan-Arctic region refers to Elven et al (2011).

极大影响了该地区的气候、环境以及生物的演化(邓 涛等, 2019; Ding et al, 2020; Li et al, 2021)。泛北极 地区植被的发生、发展主要受上新世以来气候、环 境变化的影响(Murray, 1995)。这是否意味着多倍体 物种对青藏高原高山区和泛北极地区生物多样性 的贡献不一样, 且潜在原因也不同呢? Nie等(2005) 对横断山地区已报道被子植物染色体数据进行了 初步统计, 共整理出种及种下类群552种, 发现多 倍体物种约占 $22.5 \%$; 王家坚等(2017)对青藏高原 被子植物染色体数据统计共整理出 1,001 种(包括变 种), 其中多倍体的出现频率约为 $23.0 \%$ 。Brochmann 等(2004)研究表明泛北极地区多倍体比例较高 (60.7\%), 尤其是泛北极地区特有类群的多倍体比 例在末次冰河时代冰川覆盖的大西洋区域偏高 (86.9\%)。这些研究似乎表明泛北极地区具有较高的 多倍体物种比例, 但这需要进一步证实。同时, 两 地多倍体比例差异在不同生活型的植物中是否也 呈现相似的式样尚不清楚。

本研究从文献及公共数据库中系统地收集整 理了青藏高原高山区及泛北极内已有染色体数目 报道的种子植物名录, 对所获取的数据进行了统计 分析, 探讨两地植物多倍体对其植物区系的贡献, 尤其是不同生活型的多倍体比例，以揭示两地植物 多倍体比例差异的原因。

\section{材料与方法}

\section{1 数据获取}

基于多种数据库, 包括 Chromosome Counts Database (CCDB, version 1.58; http://ccdb.tau.ac.il/)、 Index to Plant Chromosome Numbers (IPCN; http:// legacy.tropicos.org/Project/IPCN) 、 Panarctic Flora (PAF; http://panarcticflora.org/)、 Elsevier (https://www. sciencedirect.com/)、Wiley (https://onlinelibrary.wiley. com/)和中国知网(https://www.cnki.net/)等, 获取青 藏高原和泛北极地区有染色体数目报道的种子植 物种级水平上的名录和染色体倍性信息。相应地, 根据各种志书，如eFloras (http://www.efloras.org/)、 中国植物志 (http://www.iplant.cn/)等查询各个物种 的生活型信息以及青藏高原物种的海拔和生境信 息，获得每个物种的详细信息见附录1、2。

\section{2 数据处理和统计分析}

我们主要基于以下原则判断物种倍性：(1)对于 文献中有染色体基数 $(x)$ 记录的, 依据Stebbins (1950) 的定义进行倍性确定, 即植物染色体数是其所在属 染色体基数 $(x)$ 的 2 倍则为二倍体, 3 倍及 3 倍以上则 为多倍体; (2)对于染色体基数不明的, 依据文献记 载判断物种倍性, 文献中未指出倍性的物种则认为 其倍性不确定。同时, 排除虽有染色体数目报道, 但倍性难以确定的物种以及没有明确生活型信息 的物种。参考王家坚等(2017), 若某物种染色体倍性 同时具有二倍体和多倍体记录, 在分析时计为多倍 
体。作为比较, 我们也采取另外两种方式判断物种 倍性: (1)基于Grant等(1963)原则, 配子染色体数等 于或大于14即为多倍体; (2)依据Goldblatt (1980), 配子染色体数等于或大于 11 即为多倍体。

由于青藏高原地区地形环境复杂, 植被随海拔 变化垂直分布明显, 根据Körner (2003)认为“林线 是高山生物群区下界” 的观点, 我们结合生境和海 拔将青藏高原的植物分成高山和非高山物种, 即分 布在林缘及林线以上的高山灌丛、高山草甸、高山 流石滩等处的物种被视为高山物种, 分布在林线以 下的物种被视为非高山物种(附录1)。泛北极地区位 于亚、欧、北美三大洲针叶林北部边界以北, 但区 内部分物种也向南延伸到林下。为与泛北极地区形 成可比性, 我们将青藏高原分布跨越林线的物种也 归为高山物种。我们分别对青藏高原、青藏高原高 山区和泛北极地区种子植物的倍性组成和多倍体 植物的科属组成情况进行统计分析。

\section{2 结果}

\section{1 青藏高原种子植物倍性组成}

根据我们的统计, 在青藏高原具有明确倍性及 生活型信息的种子植物有 1,357 种, 隶属于 72 科 306 属, 其中木本植物 213 种 (15.7\%), 草本植物 1,144 种 (84.3\%) (附录1)。多倍体物种有299种，约占 $22.0 \%$; 二倍体物种有 1,058 种, 约占 $78.0 \%$ 。依据Grant等 (1963)和Goldblatt (1980)的多倍体界定标准, 青藏 高原内多倍体植物比例分别为 $39.2 \%$ 和 $60.4 \%$ 。青藏 高原高山区的种子植物有 774 种, 隶属于 46 科181属, 其中木本植物 47 种(6.1\%), 草本植物 727种(93.9\%) (表1，附录1)。多倍体植物物种有162种，约占20.9\%; 二倍体物种有 612 种, 约占 $79.1 \%$ (表1)。多倍体植物
中, 木本植物约占 $3.7 \%$, 草本植物约占 $96.3 \%$ (表 1)。在一年生草本、多年生草本和木本植物中多倍 体物种所占的比例分别为 $20.7 \%(18 / 87) 、 21.6 \%$ (138/640)和 $12.8 \%$ (6/47); 在一年生草本、多年生草 本和木本植物中二倍体物种所占的比例分别为 79.3\% (69/87)、78.4\% (502/640)和87.2\% (41/47) (表 1)。依据Grant等(1963)和Goldblatt (1980)的多倍体 界定方法, 青藏高原高山区的多倍体植物比例分别 为 $36.6 \%$ 和 $57.5 \%$ 。

青藏高原高山区的162种多倍体物种隶属于 26 科73属。多倍体物种数量排名前五的科为: (1)菊科, 在该区域有136种具有染色体记录, 其中多倍体物 种比例为 $23.5 \%$; (2)毛茛科, 在该区域有 116 种具有 染色体记录, 其中多倍体物种比例为 $19.0 \%$; (3)百 合科, 在该区域有 62 种具有染色体记录, 其中多倍 体物种比例为 $27.4 \%$; (4)禾本科, 在该区域有 19 种 具有染色体记录, 其中多倍体物种比例为 $68.4 \%$; (5) 虎耳草科, 在该区域有34种具有染色体记录, 其中 多倍体物种比例为 $38.2 \%$ 。多倍体物种数量排名前 五的属为: (1)香青属(Anaphalis), 在该区域有15种 具有染色体记录, 其中多倍体物种比例为 $66.7 \%$; (2) 葱属(Allium), 在该区域有23种具有染色体记录, 其 中多倍体物种比例为 $39.1 \%$; (3)龙胆属 (Gentiana), 在该区域有 57 种具有染色体记录, 其中多倍体物种 比例为 $15.8 \%$; (4)绿线蒿属(Meconopsis), 在该区域 有 9 种具有染色体记录, 其中多倍体物种比例为 88.9\%; (5)虎耳草属(Saxifraga), 在该区域有16种具 有染色体记录, 其中多倍体物种比例为 $50.0 \%$ 。

\section{2 泛北极地区种子植物倍性组成}

泛北极地区具有明确的倍性及生活型信息的 种子植物共996种, 隶属于54科299属, 其中木本植

表1 青藏高原高山区和泛北极地区种子植物倍性、生活型信息统计。百分比表示两地不同生活型及总体中的多倍体、二倍 体占比。

Table 1 Statistics of the ploidy and life form of seed plants in the Qinghai-Tibet Plateau (QTP) alpine and Pan-Arctic regions. Percentages indicate polyploid and diploid proportions of different life forms and the total in the two regions.

\begin{tabular}{llll}
\hline 生活型 Life form & $\begin{array}{l}\text { 青藏高原高山区物种数 } \\
\text { No. of species in the QTP alpine region }\end{array}$ & \multicolumn{2}{c}{$\begin{array}{l}\text { 泛北极地区物种数 } \\
\text { No. of species in the Pan-Arctic region }\end{array}$} \\
\cline { 2 - 4 } & 多倍体 Polyploid & 二倍体 Diploid & 多倍体 Polyploid \\
\hline 木本植物 Woody plant & $6(12.8 \%)$ & $41(87.2 \%)$ & $49(38.3 \%)$ \\
草本植物 Herb & $156(21.5 \%)$ & $571(78.5 \%)$ & $564(65.0 \%)$ \\
一年生草本 Annual herb & $18(20.7 \%)$ & $69(79.3 \%)$ & $53(60.2 \%)$ \\
多年生草本 Perennial herb & $138(21.6 \%)$ & $502(78.4 \%)$ & $511(65.5 \%)$ \\
总数 Total & $162(20.9 \%)$ & $612(79.1 \%)$ & $613(61.5 \%)$ \\
\hline
\end{tabular}


物128种(12.9\%), 草本植物868种(87.1\%) (附录2)。 多倍体物种有 613 种, 约占 $61.5 \%$; 二倍体物种有 383种, 约占 $38.5 \%$ (表1)。多倍体植物中, 木本植物 约占8.0\% (49/613), 草本植物约占92.0\% (564/613) (表1)。在一年生草本、多年生草本和木本植物中多 倍体物种所占的比例分别为 $60.2 \%$ (53/88)、65.5\% (511/780)和38.3\% (49/128); 在一年生草本、多年生 草本和木本植物中二倍体物种所占的比例分别为 39.8\% (35/88)、34.5\% (269/780)和61.7\% (79/128) (表 1)。依据Grant等(1963)和Goldblatt (1980)的多倍体 界定方法, 泛北极地区多倍体植物的比例分别为 $64.7 \%$ 和 $75.4 \%$ 。

泛北极地区的 613 种多倍体植物隶属于 46 科 213属。多倍体物种数量排名前五的科为: (1)禾本科, 在该区域有155种具有染色体记录, 其中多倍体物 种比例为 $80.6 \%$; (2)菊科, 在该区域有 142 种具有染 色体记录, 其中多倍体物种比例为64.8\%; (3)十字 花科, 在该区域有 89 种具有染色体记录, 其中多倍 体物种比例为 $67.4 \%$; (4)沟繁缕科, 在该区域有 70 种具有染色体记录, 其中多倍体物种比例为 $55.7 \%$; (5)石竹科, 在该区域有 60 种具有染色体记录, 其中 多倍体物种比例为 $63.3 \%$ 。多倍体物种数量排名前 五的属为: (1)早熟禾属(Poa), 在该区域有 27 种具有 染色体记录, 其中多倍体物种比例为 $88.9 \%$; (2)葶 劳属(Draba), 在该区域有27种具有染色体记录, 其 中多倍体物种比例为74.1\%; (3)棘豆属(Oxytropis), 在该区域有32种具有染色体记录, 其中多倍体物种 比例为56.3\%; (4)碱茅属(Puccinellia), 在该区域有 25 种具有染色体记录, 其中多倍体物种比例为 68.0\%; (5)柳属(Salix), 在该区域有38种具有染色体 记录, 其中多倍体物种比例为 $36.8 \%$ 。

\section{3 讨论}

3.1 青藏高原高山区和泛北极地区种子植物倍性 和生活型组成比较

王家坚等(2017)已对青藏高原被子植物染色体 数据进行了较全面的整理, 统计出具有倍性记录的 植物 1,001 种或变种。在此基础上, 我们统计出青藏 高原地区具有明确染色体倍性及生活型信息的种 子植物有 1,357 种。相对青藏高原丰富的植物多样性, 该地区已报道染色体数目、倍性的植物仍较少, 截 至目前仅约 $10 \%$, 这可能与青藏高原地区植物种类
繁多, 地形、地貌复杂, 以及野外采集困难等有关。 为了更准确地探讨多倍体物种对该地区物种多样 性的贡献, 未来应加强该地区植物细胞学的研究。

根据我们的统计, 青藏高原高山区的种子植物 多倍体比例约为 $20.9 \%$, 低于全球被子植物多倍体 的比例( 30\%-35\%; Stebbins, 1950)。我们的结果与 前人对青藏高原的研究结果相似, 如王家坚等 (2017)发现青藏高原被子植物中多倍体的比例为 23.0\%; Nie等(2005)发现横断山区被子植物中多倍 体的比例为 $22.5 \%$ 。然而, 我们的统计比例小于 Khatoon和Ali (1993)的结果(30.1\%)。这可能由于 Khatoon和Ali (1993)的统计包括了巴基斯坦许多低 海拔的非青藏高原的物种, 或者由于青藏高原内部 植物多倍体在不同区域存在一定差异。根据Grant 等(1963)和Goldblatt (1980)的倍性判断方法, 青藏 高原高山区的多倍体比例分别为 $36.6 \%$ 和 $57.5 \%$, 这 与 $\mathrm{Nie}$ 等(2005)用相同方法统计的横断山区被子植 物中的多倍体比例接近(分别为37\%和 $58 \%$ ), 并且 均小于用同一方法统计得到的全球被子植物的多 倍体比例(分别为47\%和68\%; Stebbins, 1950)。

我们统计分析显示, 泛北极地区种子植物中多 倍体的比例约为 $61.5 \%$, 这与Brochmann等(2004)对 泛北极地区多倍体比例的统计结果(60.7\%)十分接 近, 但远高于全球被子植物中多倍体的比例 ( 30\%-35\%; Stebbins, 1950)。根据Grant等(1963)和 Goldblatt (1980)的倍性判断方法, 泛北极地区的多 倍体比例分别为 $64.7 \%$ 和 $75.4 \%$, 均大于对应方法统 计得到的全球被子植物的多倍体比例(分别为 $47 \%$ 和68\%; Stebbins, 1950)。

整个青藏高原地区和泛北极地区不同植物习 性组成比例接近, 草本是 $84.3 \%$ 和 $87.1 \%$, 木本为 $15.7 \%$ 和 $12.9 \%$; 但青藏高原高山区和泛北极地区 的草本(93.9\% vs. $87.1 \%)$ 和木本(6.1\% vs. $12.9 \%)$ 植 物组成有一定差异。此外, 在多倍体物种中, 草本 植物的占比都高于木本植物: 青藏高原高山区多倍 体植物中草本和木本占比分别为 $96.3 \%$ 和 $3.7 \%$, 泛 北极地区多倍体植物中草本和木本占比分别为 $92.0 \%$ 和 $8.0 \%$ (表1)。前人研究已注意到这一现象。 例如, Stebbins (1938)发现双子叶植物中木本属多倍 体类群的发生频率低于草本属; Zenil-Ferguson等 (2017)进一步发现双子叶植物中草本植物染色体不 稳定性要远高于木本植物, 其染色体加倍的比率要 
比木本植物大6倍左右。王家坚等(2017)对青藏高原 被子植物染色体数据统计分析也发现多倍体植物 中草本比例(84.7\%)远高于木本(12.6\%)。原因可能 在于草本植物的生命周期较短, 对环境变化更加敏 感(Compagnoni, 2021), 从而导致其多倍化频率比 木本植物高。

青藏高原高山区和泛北极地区虽具有相似的 低温环境, 但我们三种判断物种倍性的方法均显示 前者种子植物多倍体的比例明显小于后者 $(20.9 \%$ vs. $61.5 \%$; $36.6 \%$ vs. $64.7 \%$; $57.5 \%$ vs. $75.4 \%)$ 。两地 不同生活型植物的多倍体比例也呈现类似的式样, 即青藏高原高山区不同生活型植物的多倍体比例 均比泛北极地区的低: 青藏高原高山区一年生草 本、多年生草本和木本植物中多倍体的比例分别为 $20.7 \% 、 21.6 \%$ 和 $12.8 \%$, 而泛北极地区一年生草本、 多年生草本和木本植物中多倍体的比例分别为 $60.2 \% 、 65.5 \%$ 和 $38.3 \%$ (表1)。同时，青藏高原高山 区多倍体数量前五的科中多倍体的比例通常也比 泛北极地区小。

\section{2 两个地区种子植物多倍体比例差异的可能原} 因

根据我们的统计结果, 青藏高原高山地区种子 植物中多倍体的比例明显低于泛北极地区, 不同生 活型植物的多倍体比例也呈现相似的式样, 这表明 两地的多倍体植物对各自种子植物区系生物多样 性的贡献是明显不同的。Rice等(2019)描述了全球 植物多倍体频率的地理分布并检验了一些关于多 倍体分布的假说, 发现影响多倍体分布的主要因素 是气候, 特别是温度。但青藏高原高山区和泛北极 两地具有相似的低温环境, 因此温度可能并非是导 致这两地多倍体比例差异的主要原因。两地多倍体 比例差异可能和两地植物区系的起源时间、地质历 史、气候事件, 以及植物多样性的来源不同有关。

\subsection{1 青藏高原高山区种子植物多倍体比例较低的} 可能原因

已有研究表明, 青藏高原高山植物区系的起源 相对较为古老。Su等(2019)对青藏高原东南缘的植 物化石研究发现, 该地植物区系于始新世末已开始 局部向现代高原植物区系过渡。Ding等(2020)针对 18 个被子植物类群的生物地理分析表明, 青藏高原 高山植物区系在早渐新世已出现(图2)。尽管青藏高 原的隆升经历了复杂多变的历史过程和极大的区
域差异, 但普遍认为渐新世-中新世之交是青藏高 原现有地形、地貌格局形成的重要阶段(王二七, 2013; 邓涛等, 2019; Su et al, 2019; Yuan et al, 2021)。青藏高原在这一时期经历了大范围的剧烈构 造活动。高原北部的祁连山、昆仑山、可可西里盆 地, 东部的峨眉山, 以及中部的伦坡拉盆地等地区 相继抬升(王二七, 2013; Ma et al, 2015)。孢粉学、 依据稳定同位素进行的古高程重建以及动植物化 石等多种证据均表明青藏高原中部在早中新世的 海拔高度已接近 $3,000 \mathrm{~m}$, 并由此产生了明显的降 温效应(Sun et al, 2014; Botsyun et al, 2019; 邓涛等, 2019; Fang et al, 2020)。高原内多数喜冷植物类群在 渐新世-中新世之交发生了快速的多样化, 原地物 种形成(in situ speciation)速率显著升高(Ding et al, 2020)。生物化石证据也表明, 高原生态系统发生了 重大转折, 植物区系在此时开始整体向现代型过渡 (图2; 邓涛等, 2019)。在中新世中期, 横断山脉和喜 马拉雅地区内类群的物种形成速率达到顶峰, 此时 受亚洲季风的影响, 地区内增加的降雨进一步增大 了地形的起伏并形成了许多新的生境(Ding et al,

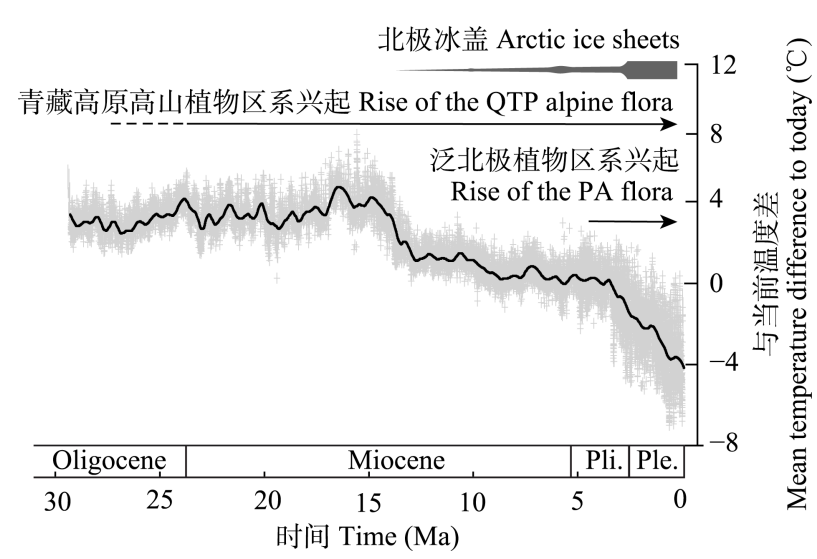

图2 青藏高原高山区和泛北极地区植物多倍体组成差异的 可能因素。QTP: 青藏高原; PA: 泛北极地区。温度曲线修 改自Westerhold等(2020)。青藏高原高山区和泛北极地区植 物区系的兴起分别依据 Ding 等 (2020) 和 Matthews 和 Ovenden (1990)的研究。虚线表示青藏高原高山植物区系在 早渐新世开始发生, 实线表示植物区系快速兴起。Oligocene: 渐新世; Miocene: 中新世; Pli.: 上新世; Ple.: 更新世。

Fig. 2 Possible factors resulting in polyploids in the QinghaiTibet Plateau (QTP) alpine and Pan-Arctic (PA) regions. The temperature curve was modified from Westerhold et al (2020). Rises of the QTP alpine and PA floras are based on Ding et al (2020) and Matthews \& Ovenden (1990), respectively. The dotted line indicates that the QTP alpine flora started to develop in the early Oligocene, whereas the solid lines indicate the rapid rise of the flora. Pli., Pliocene; Ple., Pleistocene. 
2017; Farnsworth et al, 2019), 极大地促进了生物多 样性的形成。

由于青藏高原植物区系起源较早, 受渐新世中新世之交高原抬升带来的降温效应的影响, 与现 在类似的高寒植物区系在当时已初具规模, 而后该 地区的造山运动可能并未导致物种发生剧烈的生 态位分化。青藏高原高山区的植物多样性多以本地 起源为主(Ding et al, 2020), 植物在由低海拔向上迁 移的过程中慢慢适应了环境变化, 或者在高原内邻 近高寒地区之间直接迁移而未发生明显的生态位 转变。因而, 青藏高原高山种子植物中的多倍体比 例相对较低, 二倍体物种在地区内植物多样性的演 化中可能扮演着至关重要的角色。Nie等(2005)提出, 在横断山地区多倍体植物对生物多样性未起主要 作用。杨扬和孙航(2006)以及王家坚等(2017)也都提 出多倍化可能并不是植物适应高山环境的唯一机 制。在漫长的进化时间里植物可能选择了其他进化 机制(如形态、生理方面)来适应青藏高原地区的环 境变化(施征等, 2011)。

近年来, 针对青藏高原的植物类群开展了大量 的分子系统学和生物地理学研究(Wen et al, 2014), 包括一些物种较多的大属, 如红景天属(Zhang et al, 2014)、龙胆属(Favre et al, 2016) 和虎耳草属 (Ebersbach et al, 2017)等。这些研究解析了青藏高原 地质、气候事件对其植物区系多样性起源和演化的 影响。为了确定青藏高原高山区多倍体物种的来源, 我们将染色体倍性标注在已有的系统发育树上(如 附录3A-C)。令人遗憾的是, 该地区植物的细胞学 研究相对贵乏, 绝大多数物种缺少染色体数目和/ 或倍性信息, 不能在系统发育的背景下准确推断青 藏高原的多倍体物种为本地(in situ)起源或外来 (ex situ)迁入 例如, 心叶虎耳草(Saxifraga cardiophylla) 为横断山及其邻近地区特有的多倍体物种(Tsuneo et al, 1998; Ebersbach et al, 2017), 分子系统学研究 显示其近缘物种为无斑虎耳草(S. omphalodifolia)、 玉龙虎耳草(S. forrestii)、格当虎耳草(S. gedangensis) 和滇西北虎耳草(S. dianxibeiensis)等(Ebersbach et al, 2017), 但目前这 4 个物种的染色体数据缺乏, 不能 确定多倍化事件是促进了心叶虎耳草在高原内的 物种形成还是发生在其未迁入高原之前的更早时 间 (附录3A)。相似的情况如龙胆科的西藏秦芜 (Gentiana tibetica)和类华丽龙胆 (G. sinoornata)等
(附录3B, C)。

\subsection{2 泛北极地区种子植物多倍体比例较高的可} 能原因

泛北极地区植物区系的起源时间相对较晚 (Murray, 1995), 在大约3-4 Ma, 受到格陵兰冰盖形 成带来的降温影响才开始形成(图2; Matthews \& Ovenden, 1990)。北半球在3.3 Ma左右出现第一次冰 期，之后冰期-间冰期反复波动(Miller et al, 2005; Westerhold et al, 2020), 导致物种的分布区频繁收 缩和扩张。许多基于化石证据、分子数据或物种古 分布模型等方面的研究推测, 在冰期发生时, 北半 球存在多个“避难所” (Despres et al, 2002; Michl et al, 2010; Souto et al, 2015; Chen \& Lou, 2019), 在间 冰期时这些“避难所”中的植物又重新扩散占据新的 生境。Stebbins (1984)通过对北美太平洋沿岸不同植 物区系的比较研究, 发现经历过冰期影响地区的多 倍体频率较高, 即多倍体发生频率可能与冰川作用 程度存在关联。我们的研究显示泛北极地区多倍体 的比例较高, 这与冰期-间冰期多次重复发生相关, 支持了Stebbins (1984)的观点。同时, 自上新世以来, 全球至少经历了58次海平面的快速上升(Miller et al, 2005; Woodruff \& Turner, 2009)。频繁的海平面变化 会造成海陆生态系统的替换和重组, 这使分布于泛 北极地区海陆交界地带的植物经历了快速强烈的 环境变化, 进而提高了多倍化发生的频率。此外, 泛北极地区的造山运动, 如上新世以来, 挪威斯瓦 尔巴(Svalbard)地区的地壳经历了反复的隆起和冰 川侵蚀(Lasabuda et al, 2021), 剧烈的地貌变化也可 能促使分布于泛北极地区的植物发生多倍化。

泛北极地区与青藏高原高山区相比, 除了植物 区系起源时间较晚、经历的地质气候历史不同外, 在植物区系的来源上也存在着较大的差异。一些分 子系统学研究已发现泛北极地区植物类群的来源 是多样的, 包括南部邻近地区的低地草原或湿地 (Tkach et al, 2008; Hoffmann et al, 2010); 北半球低 纬度高山, 如落基山脉(Drummond et al, 2012)、欧 洲高山系统(Comes \& Kadereit, 2003; Roquet et al, 2013)和青藏高原/喜马拉雅横断山脉 (Sun et al, 2012; Zhang et al, 2014; Favre et al, 2016)等。当全球 温度升高时, 植物类群可以由邻近的低地草原或湿 地北迁进入泛北极地区(如: Hoffmann et al, 2010); 在温度下降时, 迁入泛北极地区的物种灭绝或原地 
适应，由此造成的生态位分化可能会促使多倍化事 件的发生。来源于北半球低纬度高山的植物类群则 是在温度下降时进入低海拔地区, 而后向泛北极地 区扩散(如: Zhang et al, 2014)。这种高山来源的植物 类群已经适应了寒冷环境, 扩散到泛北极地区后可 能不需要进一步发生生态位的分化, 因而不需要发 生多倍化或者发生的频率相对较低。这一假说需要 进一步证实。泛北极地区植物的细胞学研究和染色 体倍性数据相对完善, 已有个别研究对区域内多倍 体的起源进行了初步分析(Hoffmann et al, 2010)。与 青藏高原高山区研究类似, 我们同样将染色体倍性 标注在已有的进化树上(附录3D-F), 但由于缺乏可 靠的系统树和/或祖先分布区重建，也未能确定多 倍体物种的起源地。例如, Artemisia samojedorum是 分布于泛北极地区的多倍体物种(Tkach et al, 2008), 分子系统学研究发现其近缘种为广布于亚洲的大 籽蒿(A. sieversiana; Tkach et al, 2008) (附录3D), 因 而不能确定其具体起源地。其他类似例子如蓼科的 密序大黄 (Rheum compactum) 和玄参科的兔耳草 (Lagotis glauca)等(附录3E, F)。

本研究从公共数据库中收集整理了青藏高原 高山区和泛北极两地共1,770种种子植物的染色体 数目、倍性及生活型数据。统计分析发现, 青藏高 原的种子植物多倍体比例远低于泛北极地区，表明 在两个相似的低温环境中, 多倍体植物对其植物区 系的贡献存在显著差异。这可能与青藏高原和泛北 极两地植物区系的起源时间、所经历的地质气候事 件, 以及植物多样性的来源不同相关。由于纬度差 异使两地具有不同的光周期持续时间和强度, 相应 地两地太阳辐射量也差异较大, 这些因素是否也与 两地多倍体比例差异有关, 尚需探讨。此外, 多倍 体物种尤其是泛北极地区两种不同来源的植物类 群中, 多倍体的来源是否不同仍不清楚。未来需要 整合细胞学、系统发育学、生物地理学、生态学等 多学科的研究方法对其进行深入探讨。

\section{ORCID}

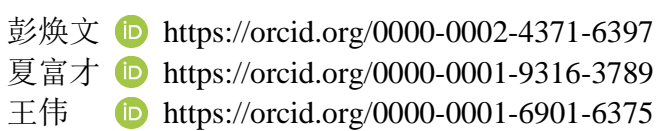

\section{参考文献}

Bennett MD (2004) Perspectives on polyploidy in
plants-Ancient and neo. Biological Journal of the Linnean Society, 82, 411-423.

Botsyun S, Sepulchre P, Donnadieu Y, Risi C, Licht A, Rugenstein JKC (2019) Revised paleoaltimetry data show low Tibetan Plateau elevation during the Eocene. Science, 363, eaaq1436.

Brochmann C, Brysting AK, Alsos IG, Borgen L, Grundt HH, Scheen AC, Elven R (2004) Polyploidy in arctic plants. Biological Journal of the Linnean Society, 82, 521-536.

Cai LM, Xi ZX, Amorim AM, Sugumaran M, Rest JS, Liu L, Davis CC (2019) Widespread ancient whole-genome duplications in Malpighiales coincide with Eocene global climatic upheaval. New Phytologist, 221, 565-576.

Chen TY, Lou AR (2019) Phylogeography and paleodistribution models of a widespread birch (Betula platyphylla Suk.) across East Asia: Multiple refugia, multidirectional expansion, and heterogeneous genetic pattern. Ecology and Evolution, 9, 7792-7807.

Comes HP, Kadereit JW (2003) Spatial and temporal patterns in the evolution of the flora of the European Alpine System. Taxon, 52, 451-462.

Compagnoni A, Levin S, Childs DZ, Harpole S, Paniw M, Römer G, Burns JH, Che-Castaldo J, Rüger N, Kunstler G, Bennett JM, Archer CR, Jones OR, Salguero-Gómez R, Knight TM (2021) Herbaceous perennial plants with short generation time have stronger responses to climate anomalies than those with longer generation time. Nature Communications, 12, 1824.

De Storme N, Geelen D (2014) The impact of environmental stress on male reproductive development in plants: Biological processes and molecular mechanisms. Plant, Cell \& Environment, 37, 1-18.

Deng T, Wu FX, Wang SQ, Su T, Zhou ZK (2019) Significant shift in the terrestrial ecosystem at the Paleogene/Neogene boundary in the Tibetan Plateau. Chinese Science Bulletin, 64, 2894-2906. (in Chinese with English abstract) [邓涛, 吴飞翔, 王世骐, 苏涛, 周浙昆 (2019) 古近纪/新近纪之 交青藏高原陆地生态系统的重大转折. 科学通报, 64, 2894-2906.]

Despres L, Loriot S, Gaudeul M (2002) Geographic pattern of genetic variation in the European globeflower Trollius europaeus L. (Ranunculaceae) inferred from amplified fragment length polymorphism markers. Molecular Ecology, 11, 2337-2347.

Ding L, Spicer RA, Yang J, Xu Q, Cai F, Li S, Lai Q, Wang H, Spicer TEV, Yue Y, Shukla A, Srivastava G, Khan MA, Bera S, Mehrotra R (2017) Quantifying the rise of the Himalaya orogen and implications for the South Asian monsoon. Geology, 45, 215-218.

Ding WN, Ree RH, Spicer RA, Xing YW (2020) Ancient orogenic and monsoon-driven assembly of the world's richest temperate alpine flora. Science, 369, 578-581.

Drummond CS, Eastwood RJ, Miotto STS, Hughes CE (2012) 
Multiple continental radiations and correlates of diversification in Lupinus (Leguminosae): Testing for key innovation with incomplete taxon sampling. Systematic Biology, 61, 443-460.

Ebersbach J, Muellner-Riehl AN, Michalak I, Tkach N, Hoffmann MH, Röser M, Sun H, Favre A (2017) In and out of the Qinghai-Tibet Plateau: Divergence time estimation and historical biogeography of the large arctic-alpine genus Saxifraga L. Journal of Biogeography, 44, 900-910.

Elven R, Murray DF, Razzhivin VY, Yurtsev BA (2011) Annotated Checklist of the Panarctic Flora (PAF), Vascular Plants. http://Panarcticflora.org/. (accessed on 2020-10-20)

Fang XM, Dupont-Nivet G, Wang CS, Song CH, Meng QQ, Zhang WL, Nie JS, Zhang T, Mao ZQ, Chen Y (2020) Revised chronology of central Tibet uplift (Lunpola Basin). Science Advances, 6, eaba7298.

Farnsworth A, Lunt DJ, Robinson SA, Valdes PJ, Roberts WHG, Clift PD, Markwick P, Su T, Wrobel N, Bragg F, Kelland SJ, Pancost RD (2019) Past East Asian monsoon evolution controlled by paleogeography, not $\mathrm{CO}_{2}$. Science Advances, 5, eaax1697.

Favre A, Michalak I, Chen CH, Wang JC, Pringle JS, Matuszak S, Sun H, Yuan YM, Struwe L, Muellner-Riehl AN (2016) Out-of-Tibet: The spatio-temporal evolution of Gentiana (Gentianaceae). Journal of Biogeography, 43, 1967-1978.

Gamberg M (2020) Threats to arctic ecosystems. In: Encyclopedia of the World's Biomes (eds Goldstein MI, DellaSala DA), pp. 532-538. Elsevier, Oxford.

Gao QZ, Guo YQ, Xu HM, Ganjurjav H, Li Y, Wan YF, Qin XB, Ma X, Liu S (2016) Climate change and its impacts on vegetation distribution and net primary productivity of the alpine ecosystem in the Qinghai-Tibetan Plateau. Science of the Total Environment, 554/555, 34-41.

Goldblatt P (1980) Polyploidy in angiosperms: Monocotyledons. In: Polyploidy: Biological Relevance (ed. Lewis WH), pp. 219-239. Springer, New York.

Grant JK, De DC, Biochimie SBD (1963) Methods of Separation of Subcellular Structural Components. Cambridge University Press, Cambridge.

Hoffmann MH, von Hagen KB, Hörandl E, Röser M, Tkach NV (2010) Sources of the arctic flora: Origins of arctic species in Ranunculus and related genera. International Journal of Plant Sciences, 171, 90-106.

Hou Y, Nowak MD, Mirré V, Bjorå CS, Brochmann C, Popp M (2016) RAD-seq data point to a northern origin of the arctic-alpine genus Cassiope (Ericaceae). Molecular Phylogenetics and Evolution, 95, 152-160.

Khatoon S, Ali SI (1993) Chromosome Atlas of the Angiosperms of Pakistan. BCC \& T Press, Karachi.

Körner C (2003) The alpine life zone. In: Alpine Plant Life: Functional Plant Ecology of High Mountain Ecosystems (ed. Körner C), pp. 9-20. Springer-Verlag, New York.

Lasabuda APE, Johansen NS, Laberg JS, Faleide JI, Senger K,
Rydningen TA, Patton H, Knutsen S-M, Hanssen A (2021) Cenozoic uplift and erosion of the Norwegian Barents Shelf -A review. Earth-Science Reviews, 217, 103609.

Levin DA (2002) The Role of Chromosomal Change in Plant Evolution (Oxford Series in Ecology and Evolution). Oxford University Press, New York.

Li GD, Kim C, Zha HG, Zhou Z, Nie ZL, Sun H (2014) Molecular phylogeny and biogeography of the arctic-alpine genus Lagotis (Plantaginaceae). Taxon, 63, 103-115.

Li SF, Valdes PJ, Farnsworth A, Davies-Barnard T, Su T, Lunt DJ, Spicer RA, Liu J, Deng WYD, Huang J, Tang H, Ridgwell A, Chen LL, Zhou ZK (2021) Orographic evolution of northern Tibet shaped vegetation and plant diversity in eastern Asia. Science Advances, 7, eabc7741.

Liu XD, Chen BD (2000) Climatic warming in the Tibetan Plateau during recent decades. International Journal of Climatology, 20, 1729-1742.

Ma PF, Wang LC, Wang CS, Wu XH, Wei YS (2015) Organic-matter accumulation of the lacustrine Lunpola oil shale, central Tibetan Plateau: Controlled by the paleoclimate, provenance, and drainage system. International Journal of Coal Geology, 147/148, 58-70.

Masterson J (1994) Stomatal size in fossil plants: Evidence for polyploidy in majority of angiosperms. Science, 264, 421424.

Matthews JV, Ovenden LE (1990) Late Tertiary plant macrofossils from localities in arctic/subarctic North America: A review of the data. ARCTIC, 43, 364-392.

Michl T, Huck S, Schmitt T, Liebrich A, Haase P, Büdel B (2010) The molecular population structure of the tall forb Cicerbita alpina (Asteraceae) supports the idea of cryptic glacial refugia in central Europe. Botanical Journal of the Linnean Society, 164,142-154.

Miller KG, Kominz MA, Browning JV, Wright JD, Mountain GS, Katz ME, Sugarman PJ, Cramer BS, Christie-Blick N, Pekar SF (2005) The Phanerozoic record of global sea-level change. Science, 310, 1293-1298.

Murray DF (1995) Causes of arctic plant diversity: Origin and evolution. In: Arctic and Alpine Biodiversity: Patterns, Causes and Ecosystem Consequences (eds Chapin FS, Korner C), pp. 21-32. Springer, Heidelberg, Germany.

Myers-Smith IH, Elmendorf SC, Beck PSA, Wilmking M, Hallinger M, Blok D, Tape KD, Rayback SA, Macias-Fauria M, Forbes BC (2015) Climate sensitivity of shrub growth across the tundra biome. Nature Climate Change, 5, 887-891.

Nie ZL, Wen J, Gu ZJ, Boufford DE, Sun H (2005) Polyploidy in the flora of the Hengduan Mountains hotspot, Southwestern China. Annals of the Missouri Botanical Garden, 92, 275306.

Pan BT, Gao HS, Li BY, Li JJ (2004) Step-like landforms and uplift of the Qinghai-Xizang Plateau. Quaternary Sciences, 24, 50-57, 133. (in Chinese with English abstract) [潘保田, 高红山, 李炳元, 李吉均 (2004) 青藏高原层状地貌与高 
原隆升. 第四纪研究, 24, 50-57, 133.]

Rice A, Šmarda P, Novosolov M, Drori M, Glick L, Sabath N, Meiri S, Belmaker J, Mayrose I (2019) The global biogeography of polyploid plants. Nature Ecology \& Evolution, 3, 265-273.

Roquet C, Boucher FC, Thuiller W, Lavergne S (2013) Replicated radiations of the alpine genus Androsace (Primulaceae) driven by range expansion and convergent key innovations. Journal of Biogeography, 40, 1874-1886.

Serreze MC, Barry RG (2011) Processes and impacts of Arctic amplification: A research synthesis. Global and Planetary Change, 77, 85-96.

Shi Z, Bai DZ, Lei JP, Li MH, Xiao WF (2011) Advance on physioecological adaptation of alpine plants to mountainous environment. Acta Botanica Boreali-Occidentalia Sinica, 31, 1711-1718. (in Chinese with English abstract) [施征, 白登 忠, 雷静品, 李迈和, 肖文发 (2011) 高山植物对其环境 的生理生态适应性研究进展. 西北植物学报, 31, 17111718.]

Souto CP, Kitzberger T, Arbetman MP, Premoli AC (2015) How do cold-sensitive species endure ice ages? Phylogeographic and paleodistribution models of postglacial range expansion of the mesothermic drought-tolerant conifer Austrocedrus chilensis. New Phytologist, 208, 960-972.

Spicer RA, Su T, Valdes PJ, Farnsworth A, Wu FX, Shi GL, Spicer TEV, Zhou ZK (2021) Why 'the uplift of the Tibetan Plateau' is a myth. National Science Review, 8, nwaa091.

Stebbins GL (1938) Cytological characteristics associated with the different growth habits in the dicotyledons. American Journal of Botany, 25, 189-198.

Stebbins GL (1950) Variation and Evolution in Plants. Columbia University Press, New York.

Stebbins GL (1984) Polyploidy and the distribution of the arctic-alpine flora: New evidence and a new approach. Botanica Helvetica, 94, 1-13.

Su T, Farnsworth A, Spicer RA, Huang J, Wu FX, Liu J, Li SF, Xing YW, Huang YJ, Deng WYD, Tang H, Xu CL, Zhao F, Srivastava G, Valdes PJ, Deng T, Zhou ZK (2019) No high Tibetan Plateau until the Neogene. Science Advances, 5, eaav2189.

Su T, Spicer RA, Li SH, Xu H, Huang J, Sherlock S, Huang YJ, Li SF, Wang L, Jia LB, Deng WYD, Liu J, Deng CL, Zhang ST, Valdes PJ, Zhou ZK (2019) Uplift, climate and biotic changes at the Eocene-Oligocene transition in south-eastern Tibet. National Science Review, 6, 495-504.

Sun JM, Xu QH, Liu WM, Zhang ZQ, Xue L, Zhao P (2014) Palynological evidence for the latest Oligocene Early Miocene paleoelevation estimate in the Lunpola Basin, central Tibet. Palaeogeography, Palaeoclimatology, Palaeoecology, 399, 21-30.

Sun YS, Wang AL, Wan DS, Wang Q, Liu JQ (2012) Rapid radiation of Rheum (Polygonaceae) and parallel evolution of morphological traits. Molecular Phylogenetics and Evolution,
63, 150-158.

Tkach NV, Hoffmann MH, Röser M, von Hagen KB (2008) Temporal patterns of evolution in the Arctic explored in Artemisia L. (Asteraceae) lineages of different age. Plant Ecology \& Diversity, 1, 161-169.

Tsuneo F, Katsuhiko K, Hong DY, Zhou SL, Takuko S (1998) A karyomorphological comparison of four Saxifraga species collected in the western part of Sichuan Province, China. Chromosome Science, 2, 103-109.

Van de Peer Y, Ashman TL, Soltis PS, Soltis DE (2021) Polyploidy: An evolutionary and ecological force in stressful times. The Plant Cell, 33, 11-26.

Wang EQ (2013) Evolution of the Tibetan Plateau: As constrained by major tectonic-thermo events and a discussion on their origin. Chinese Journal of Geology, 48, 334-353. (in Chinese with English abstract) [王二七 (2013) 青藏高原大地构造演化一一主要构造-热事件的 制约及其成因探讨. 地质科学, 48, 334-353.]

Wang JJ, Peng ZB, Sun H, Nie ZL, Meng Y (2017) Cytogeographic patterns of angiosperms flora of the QinghaiTibet Plateau and Hengduan Mountains. Biodiversity Science, 25, 218-225. (in Chinese with English abstract) [王 家坚, 彭智邦, 孙航, 聂泽龙, 孟盈 (2017) 青藏高原与 横断山被子植物区系演化的细胞地理学特征. 生物多样 性, 25, 218-225.]

Wang Q, Liu JQ, Allen GA, Ma YZ, Yue W, Marr KL, Abbott RJ (2016) Arctic plant origins and early formation of circumarctic distributions: A case study of the mountain sorrel, Oxyria digyna. New Phytologist, 209, 343-353.

Wang YF, Sylvester SP, Lu XM, Dawadi B, Sigdel SR, Liang EY, Julio Camarero J (2019) The stability of spruce treelines on the eastern Tibetan Plateau over the last century is explained by pastoral disturbance. Forest Ecology and Management, 442, 34-45.

Wen J, Zhang JQ, Nie ZL, Zhong Y, Sun H (2014) Evolutionary diversifications of plants on the QinghaiTibetan Plateau. Frontiers in Genetics, 5, 4.

Westerhold T, Marwan N, Drury AJ, Liebrand D, Agnini C, Anagnostou E, Barnet JSK, Bohaty SM, De Vleeschouwer D, Florindo F, Frederichs T, Hodell DA, Holbourn AE, Kroon D, Lauretano V, Littler K, Lourens LJ, Lyle M, Pälike H, Röhl U, Tian J, Wilkens RH, Wilson PA, Zachos JC (2020) An astronomically dated record of Earth's climate and its predictability over the last 66 million years. Science, 369, 1383-1387.

Woodruff DS, Turner LM (2009) The Indochinese-Sundaic zoogeographic transition: A description and analysis of terrestrial mammal species distributions. Journal of Biogeography, 36, 803-821.

Wu SD, Han BC, Jiao YN (2020) Genetic contribution of paleopolyploidy to adaptive evolution in angiosperms. Molecular Plant, 13, 59-71.

Yang Y, Sun H (2006) Advances in the functional ecology of 
alpine and arctic plants. Acta Botanica Yunnanica, 28, 43-53. (in Chinese with English abstract) [杨扬, 孙航 (2006) 高山和极地植物功能生态学研究进展. 云南植物 研究, 28, 43-53.]

Yuan J, Yang ZY, Deng CL, Krijgsman W, Hu XM, Li SH, Shen ZS, Qin HF, An W, He HY, Ding L, Guo ZT, Zhu RX (2021) Rapid drift of the Tethyan Himalaya terrane before two-stage India-Asia collision. National Science Review, 8, nwaa173.

Zenil-Ferguson R, Ponciano JM, Burleigh JG (2017) Testing the association of phenotypes with polyploidy: An example using herbaceous and woody eudicots. Evolution, 71, 11381148.

Zhang DC, Ye JX, Sun H (2016) Quantitative approaches to identify floristic units and centres of species endemism in the Qinghai-Tibetan Plateau, south-western China. Journal of Biogeography, 43, 2465-2476.

Zhang JQ, Meng SY, Allen GA, Wen J, Rao GY (2014) Rapid radiation and dispersal out of the Qinghai-Tibetan Plateau of an alpine plant lineage Rhodiola (Crassulaceae). Molecular Phylogenetics and Evolution, 77, 147-158.

Zhang YL, Li BY, Zheng D (2002) A discussion on the boundary and area of the Tibetan Plateau in China. Geographical Research, 21, 1-8. (in Chinese with English abstract) [张镱锂, 李炳元, 郑度 (2002) 论青藏高原范围 与面积. 地理研究, 21, 1-8.]

(责任编委: 高连明 责任编辑: 时意专)

\section{附录 Supplementary Material}

附录1 青藏高原种子植物倍性、生活型、海拔和生境

Appendix 1 Ploidy, life form, elevation, and habitat of seed plants in the Qinghai-Tibet Plateau https://www.biodiversity-science.net/fileup/PDF/2021146-1.xlsx

\section{附录2 泛北极地区种子植物倍性及生活型}

Appendix 2 Ploidy and life form of seed plants in the Pan-Arctic region https://www.biodiversity-science.net/fileup/PDF/2021146-2.xlsx

\section{附录3 在系统发育框架下分析植物多倍体的起源}

Appendix 3 The origin of a few polyploids analyzed in a phylogenetic context https://www.biodiversity-science.net/fileup/PDF/2021146-3.pdf 
张军, 彭焕文, 夏富才, 王伟 (2021) 青藏高原高山区和泛北极地区种子植物多倍体比较. 生物多样性, 29, 1470-1480. http://www.biodiversity-science.net/CN/10.17520/biods.2021146
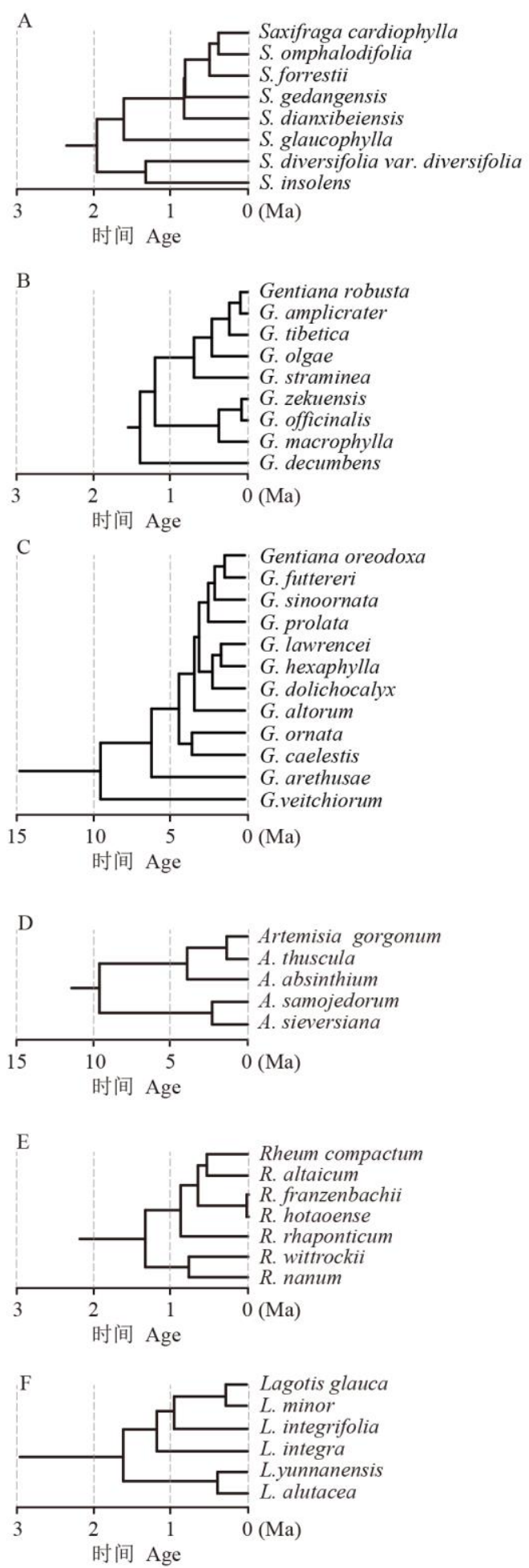

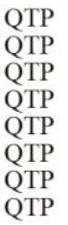

QTP

QTP

QTP

QTP

QTP

QTP

Eastern Asia

Europ, Northern Asia, QTP, Eastern Asia

QTP

QTP

QTP

QTP

QTP

QTP

QTP

QTP

QTP

QTP

QTP

$\begin{array}{ll}2 x & \text { Africa } \\ 2 x & \text { Africa } \\ 2 x, 4 x & \text { Asia, Europe, Africa } \\ 6 x & \text { Pan-Arctic } \\ 2 x & \text { Asia }\end{array}$

Pan-Aretic

Asia
Pan-Arctic, Central Asian Central Asian QTP

QTP

Europe

Central Asian

Central Asian

附录3 在系统发育框架下分析植物多倍体的起源。A，虎耳草属(Saxifraga), 时间树源自Ebersbach等 (2017); B、C, 龙胆属 (Gentiana)，时间树源自Favre等(2016); D, 蒿属(Artemisia), 时间树源自Tkach等 (2008); E，大黄属 (Rheum), 时间树源自Sun 等(2012); F, 兔耳草属(Lagotis)，时间树源自 Li等(2014)。“?”示缺乏染色体数据。

Appendix 3 The origin of a few polyploids analyzed in a phylogenetic context. A, Saxifraga, timetree was modified from Ebersbach et al (2017); B and C, Gentiana, timetree was modified from Favre et al (2016); D, Artemisia, timetree was modified from Tkach et al (2008); E, Rheum, timetree was modified from Sun et al (2012); F, Lagotis, timetree was modified from Li et al (2014).

"?" indicates that chromosome data are missing.

Pan-Arctic, Central Asian
Central Asian
QTP
QTP
QTP
QTP

